\title{
Depletion of ZBTB38 potentiates the effects of DNA demethylating agents in cancer cells via CDKN1C mRNA up-regulation
}

\author{
Claire Marchal 1,2,3,7, Maud de Dieuleveult ${ }^{1,2,3}$, Claude Saint-Ruf ${ }^{1,2,3}$, Nadège Guinot ${ }^{1,2,3}$, Laure Ferry ${ }^{4}$, Sara T. Olalla Saad ${ }^{5}$,
} Mariana Lazarini, ${ }^{6}$ Pierre-Antoine Defossez (10 ${ }^{4}$ and Benoit Miotto ${ }^{1,2,3}$

\begin{abstract}
DNA methyltransferase inhibitor (DNMTi) treatments have been used for patients with myelodysplastic syndromes (MDS) and acute myeloid leukemia (AML), and have shown promising beneficial effects in some other types of cancers. Here, we demonstrate that the transcriptional repressor ZBTB38 is a critical regulator of the cellular response to DNMTi. Treatments with 5-azacytidine, or its derivatives decitabine and zebularine, lead to down-regulation of ZBTB38 protein expression in cancer cells, in parallel with cellular damage. The depletion of ZBTB38 by RNA interference enhances the toxicity of DNMTi in cell lines from leukemia and from various solid tumor types. Further we observed that inactivation of ZBTB38 causes the up-regulation of CDKN1C MRNA, a previously described indirect target of DNMTi. We show that CDKN1C is a key actor of DNMTi toxicity in cells lacking ZBTB38. Finally, in patients with MDS a high level of CDKN1C mRNA expression before treatment correlates with a better clinical response to a drug regimen combining 5-azacytidine and histone deacetylase inhibitors. Collectively, our results suggest that the ZBTB38 protein is a target of DNMTi and that its depletion potentiates the toxicity of DNMT inhibitors in cancer cells, providing new opportunities to enhance the response to DNMT inhibitor therapies in patients with MDS and other cancers.
\end{abstract}

\section{Introduction}

Vidaza (5-azacytidine), decitabine (5-aza-2-deoxy-cytidine), and zebularine (2(1 H)-pyrimidinone riboside) belong to a class of cytosine analogues that were developed as inhibitors of DNA methylation. The incorporation of these analogues into the DNA (and/or RNA) leads to the formation of covalent bond between the nucleoside analogue and the cysteine thiolate in the catalytic site of the DNA methyltransferases (DNMTs) that establish and maintain DNA methylation patterns during development. This phenomenon eventually leads to the sequestration of the DNMTs, their depletion in the cell, and the passive

Correspondence: Benoit Miotto (benoit.miotto@inserm.fr)

${ }^{1}$ INSERM, U1016, Institut Cochin, Paris, France

${ }^{2}$ CNRS, UMR8104, Paris, France

Full list of author information is available at the end of the article.

These authors contributed equally: Claire Marchal, Maud de Dieuleveult demethylation of the genomic DNA during DNA replication $^{1-4}$.

5-azacytidine and decitabine have been used to improve survival and health quality of patients with myelodysplastic syndromes (MDS), acute myelogenous leukemia (AML) and chronic myelomonocytic leukemia (CMML) ${ }^{4-}$ ${ }^{6}$. Nonetheless, due to their incorporation into the DNA and the formation of DNA adducts these drugs may have unwanted side effects, limiting their clinical applications ${ }^{4,7}$. There is thus need to develop new therapeutic strategies (i.e., new DNMT inhibitors) and to identify biomarkers that may help predict which patient will most benefit from DNMTi therapy. Several genetic studies have shown that the toxicity and the clinical response of 5azacytidine derivatives in patients with MDS and AML is influenced by the genetic context ${ }^{8,9}$. Mutations in TP53, TET2, IDH1, IDH2, GADD45A, and DNMT3A correlate with better or poorer drug response in MDS and AML

\section{(c) The Author(s) 2018}

(c) (i) Open Access This article is licensed under a Creative Commons Attribution 4.0 International License, which permits use, sharing, adaptation, distribution and reproduction cc) in any medium or format, as long as you give appropriate credit to the original author(s) and the source, provide a link to the Creative Commons license, and indicate if changes were made. The images or other third party material in this article are included in the article's Creative Commons license, unless indicated otherwise in a credit line to the material. If material is not included in the article's Creative Commons license and your intended use is not permitted by statutory regulation or exceeds the permitted use, you will need to obtain permission directly from the copyright holder. To view a copy of this license, visit http://creativecommons.org/licenses/by/4.0/. 
patients $^{10-17}$. At the transcriptional level, expression of PLBC1，BCL2L10 or micro-RNA-126 influence the response to DNMTi ${ }^{18-20}$. Furthermore, the efficacy of 5azacytidine can be further enhanced by combination with other compounds including histone deacetylase inhibitors $(\text { HDACi })^{1,4,7,21}$.

The reasons of the toxicity, as well as the mechanism of action of DNMTi, remain not yet fully understood.

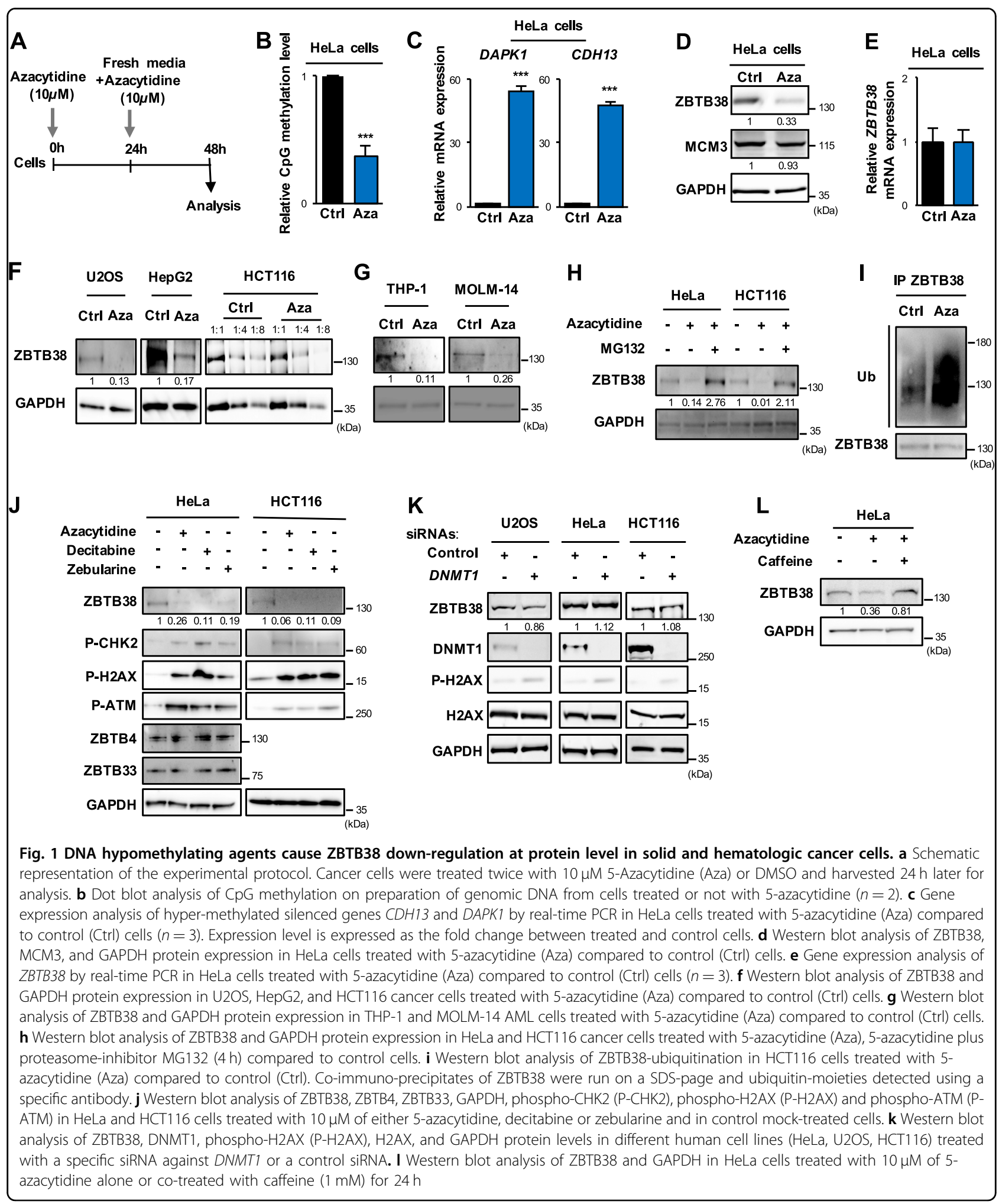


DNMTi cause passive demethylation of the genomic DNA during DNA replication, coincident with cell proliferation defects and changes in gene expression ${ }^{2,3,22,23}$. Yet, different DNMT inhibitors have variable impact on gene expression, cellular processes and cell death on similar tumor types, questioning the existence of additional effects on protein synthesis, chromatin structure regulation and cell death pathways ${ }^{3,14,21-23}$. For instance, depletion of transcription factor p53 in embryonic fibroblasts from mice strongly enhances the cytotoxicity of 5azacytidine treatments by potentiating a deadly interferon response $^{24}$. A similar phenomenon has been documented in human ovarian cancer cells exposed to decitabine ${ }^{15,25}$.

Herein, we hypothesized that DNMTi might have an effect on the transcription factors that bind methylated DNA, so we evaluated the impact of 5-azacytidine on the function and expression of the zinc finger and BTB domain containing protein ZBTB38, that binds to methyl$\mathrm{CpGs}^{26-28}$. ZBTB38 is involved in various cellular functions, including the regulation of DNA replication, the control of gene expression and the regulation of cell proliferation and differentiation ${ }^{26,29-32}$. We observed that 5 -azacytidine causes the down-regulation of ZBTB38 protein expression. In addition, we demonstrated that the depletion of ZBTB38, or its regulator deubiquitinase USP9X, enhances the cytotoxicity of 5-azacytidine derivatives in different cancer and leukemia cells, which was concomitant with the enhanced expression of CDKN1C mRNA. Finally, we observed a correlation between CDKN1C mRNA expression in MDS patients and the clinical response to a combination of 5-azacytidine and HDACi. Altogether our work suggests that inhibition (or inactivation) of ZBTB38 or USP9X expression may be a new strategy to enhance the clinical efficacy of DNMTi in hematological and non-hematological cancers.

\section{Results \\ 5-azacytidine causes a decrease of ZBTB38 protein \\ abundance}

Transcription factor ZBTB38 binds with high affinity to DNA sequences containing methylated $\mathrm{CpG}$ sites in vitro, and is recruited at hyper-methylated peri-centromeric sequences in murine cells ${ }^{27-30,33}$. We thus decided to further explore the relationship between ZBTB38 and DNA methylation and tested whether alteration of DNA methylation pattern would interfere with the function of ZBTB38. We exposed human HeLa cells to 5-azacytidine for $48 \mathrm{~h}$ (Fig. 1a), which led to global loss of CpG methylation (Fig. 1b). We further confirmed the loss of methylation by showing that hyper-methylated genes (CDH13 and DAPK1) were highly expressed in 5azacytidine treated samples compared to control samples (Fig. 1c). Western blot analysis showed that ZBTB38 expression was lower in 5 -azacytidine-treated whole cell protein extracts compared to untreated cells (Fig. 1d). Expression of the DNA replication factor MCM3 was used as a negative control and excluded a general impact of 5-azacytidine on the activity of the proteasome or on mechanisms of protein synthesis (Fig. 1d). Differing from protein expression, ZBTB38 mRNA was expressed at similar levels in 5-azacytidine-treated cells compared to control cells (Fig. 1e). In three additional human cancer types (U2OS, HepG2, and HCT116) and two leukemia cell types (THP-1 and MOLM-14) we also observed that exposure to 5-azacytidine causes the down-regulation of ZBTB38 protein abundance without altering the mRNA level (Fig. 1f, g and Supplementary Fig. S1A, B). The amplitude of ZBTB38 protein down-regulation is variable among the cell types studied (Fig. 1d, f, g). We thus monitored the level of expression of ZBTB38 protein in a set of isogenic cells: HCT116 DNMT1 and DNMT3B knockout (HCT116 DKO), HCT116 TP53 ${ }^{-1-}$ and parental HCT116 cells treated with 5-azacytidine (Supplementary Fig. S1C, D). In all cell types, 5-azacytidine treatment induced a dose-dependent down-regulation of ZBTB38 protein, but the down-regulation in HCT116 DKO cells was rather modest while it was much stronger in HCT116 TP53 ${ }^{-1-}$ cells compared to isogenic HCT116 cells (Supplementary Fig. S1C, D). We then investigated the abundance of ZBTB38 in MOLM14 cells (i.e., AML cells p53 positive) transfected with siRNA against p53 or control siRNA and further exposed to azacytidine. The abundance of ZBTB38 was lower in cells transfected with siRNAs against p53 (Supplementary Fig. S1E). In other words, 5-azacytidine causes the down regulation of ZBTB38 protein expression in a variety of cancer and leukemia cells and this regulation is affected by the cellular background of the cell, notably the status of DNMT1/3B and TP53.

ZBTB38 is regulated by poly-ubiquitination and proteasome degradation ${ }^{30-32}$. We thus investigated the implication of this pathway in 5-azacytidine induced ZBTB38 protein down-regulation. For this purpose, HeLa and HCT116 cells were concomitantly treated with a proteasome inhibitor (MG132) and 5-azacytidine. We observed that the addition of MG132 induced a higher ZBTB38 expression in comparison to 5-azacytidine alone (Fig. 1h). Further, we immunoprecipitated ZBTB38 from HCT116 cells treated with 5-azacytidine (in presence of MG132). We confirmed that ZBTB38 is polyubiquitinated in control cells and observed that the amount of poly-ubiquitination is dramatically increased in 5-azacytidine treated cells (Fig. 1i). In conclusion, 5azacytidine causes ZBTB38 down-regulation in different cancer cell types, mainly at the protein level and most likely in a proteasome-dependent manner. We therefore investigated the regulation of E3 ubiquitin ligase RBBP6 and deubiquitinase USP9X that controls ZBTB38 poly- 
ubiquitination in human cells $\mathrm{s}^{31,32}$. We observed that RBBP6 protein and mRNA levels were up-regulated upon 5-azacytidine treatment and that this up-regulation occurs at the protein and mRNA levels (Supplementary Fig. S1F, G). No detectable changes in USP9X protein levels were observed at the time point tested (Supplementary Fig. $\mathrm{S} 1 \mathrm{H}$ ). We tested the hypothesis that RBBP6 up-regulation may be a consequence of the demethylation of RBBP6 promoter. Using immunoprecipitation of methylated DNA, we could not detect significant levels of DNA methylation at the RBBP6 promoter (Supplementary Fig. S1I), arguing against a direct effect of 5azacytidine on the methylation of the RBBP6 promoter. These data indicate that the down-regulation of ZBTB38 protein levels upon 5-azacytidine treatment is mediated by an ubiquitination pathway and most likely occurs through the up-regulation of E3 ubiquitin ligase RBBP6.

\section{Down-regulation of ZBTB38 protein level coincides with 5- azacytidine cytotoxicity}

Azacytidine mechanism of action is associated with its DNA demethylating activity but also with indirect cytotoxic effects ${ }^{2,15,22-25,34}$. We thus monitored ZBTB38 protein expression in three conditions: in cells treated with different DNMT inhibitors, in cells genetically inactivated for the enzymes involved in the establishment and maintenance of DNA methylation and in cells transfected with DNMT1 RNAi molecules. In parallel, we monitored DNA methylation levels and the presence of DNA damage in the same samples.

Azacytidine, decitabine, and zebularine treated cells presented decreased ZBTB38 protein expression compared to control samples (Fig. 1j), while ZBTB38 mRNA levels were quite similar between the different samples (Supplementary Fig. S1J). Again, the effect of DNMTi was specific as the expression of ZBTB4 and ZBTB33/KAISO, two paralogs of ZBTB38, was not altered (Fig. 1j). We observed a significant increase in phospho-H2AX, a marker of cellular damage, in DNMTi treated cells compared to vehicle treated cells (Fig. 1j). Cellular damage was confirmed by the increase in phospho-CHK2 and phospho-ATM signals in the same DNMTi treated samples (Fig. 1j). Importantly, cells genetically inactivated for DNMT1 and DNMT3B (HCT116 Double Knock-Out), which lose $\sim 75 \%$ of their DNA methylation, did not present changes in ZBTB38 protein abundance compared to isogenic parental cells (Supplementary Fig. S1K, L). This observation indicates that ZBTB38 protein expression was not directly correlated with the steady-state amount of DNA methylation in cells, nor dependent on a physical interaction with DNMT1 or DNMT3B.

DNMT1 is the main enzyme that restores CpG methylation patterns following genome replication, so we investigated the consequence of its depletion on ZBTB38 protein abundance. DNMT1 silencing was confirmed by western blot after $48 \mathrm{~h}$ of transfection in HeLa, U2OS, and HCT116 cells with specific siRNA directed against DNMT1 (Fig. 1k) and we confirmed that heavily methylated and silenced tumor suppressor genes were reactivated (Supplementary Fig. S2). ZBTB38 protein expression level was similar between DNMT1-depleted and control cells (Fig. 1k). In addition, we observed that H2AX phosphorylation was only barely increased in cells transfected with siRNAs against DNMT1 compared to control cells (Fig. 1k). From this set of experiments, we concluded that the lack of DNMT1 (or its strong reduction by RNA interference), accompanied by a loss of DNA methylation, does not alter ZBTB38 protein expression. These results indicate that ZBTB38 protein downregulation by DNMT inhibitors is most likely a consequence of the activation of a stress response pathway or cell death promotion. We thus investigated the level of expression of ZBTB38 in HeLa cells combining DNMTi treatment with caffeine, an inhibitor of ATM-ATR damage-induced kinases. Consistent with our interpretation, we observed that the level of ZBTB38 in cells cotreated with caffeine is significantly higher than in cells treated with azacytidine alone (Fig. 11).

\section{Depletion of ZBTB38 does not increase DNA demethylation by DNMT inhibitors}

We speculated that DNMTi might achieve a higher DNA demethylation in the absence of ZBTB38 protein. To test this hypothesis, we characterized the extent of DNA demethylation induced by DNMTi in ZBTB38silenced cells. The amount of 5-methyl-cytosine $(5-\mathrm{mC})$ and 5-hydroxymethylcytosine (5-hmC), an intermediate in DNA demethylation, was determined on genomic DNA preparations by dot blot. We observed that levels of 5-mC and 5-hmC were similar in cells transfected with control and ZBTB38 siRNAs as well as in cells combining RNA interference and 5-azacytidine (or decitabine) treatment (Fig. 2a-c). Therefore, depletion of ZBTB38 by RNA interference does not potentiate the demethylating activity of DNMTi in the tested conditions at the global scale.

We further investigated the expression of enzymes involved in the regulation of DNA methylation, whose mutation and/or expression level were reported to influence 5-azacytidine derivatives sensitivity in AML and MDS patients ${ }^{10-12,16,17,35}$. We evaluated the expression of DNMT1, DNMT3A, TET2, GADD45A, UCK1, IDH1 and IDH2 in HCT116, U2OS and THP-1 cells transfected with control and ZBTB38 siRNAs (Fig. 2d). All of these genes were expressed at very similar levels in ZBTB38 depleted cells and control cells (Fig. 2d). In conclusion, depletion of ZBTB38 does not potentiate the DNA demethylating activity of 5-azacytidine and decitabine, nor does it affect the mRNA expression of the tested set of genes involved 


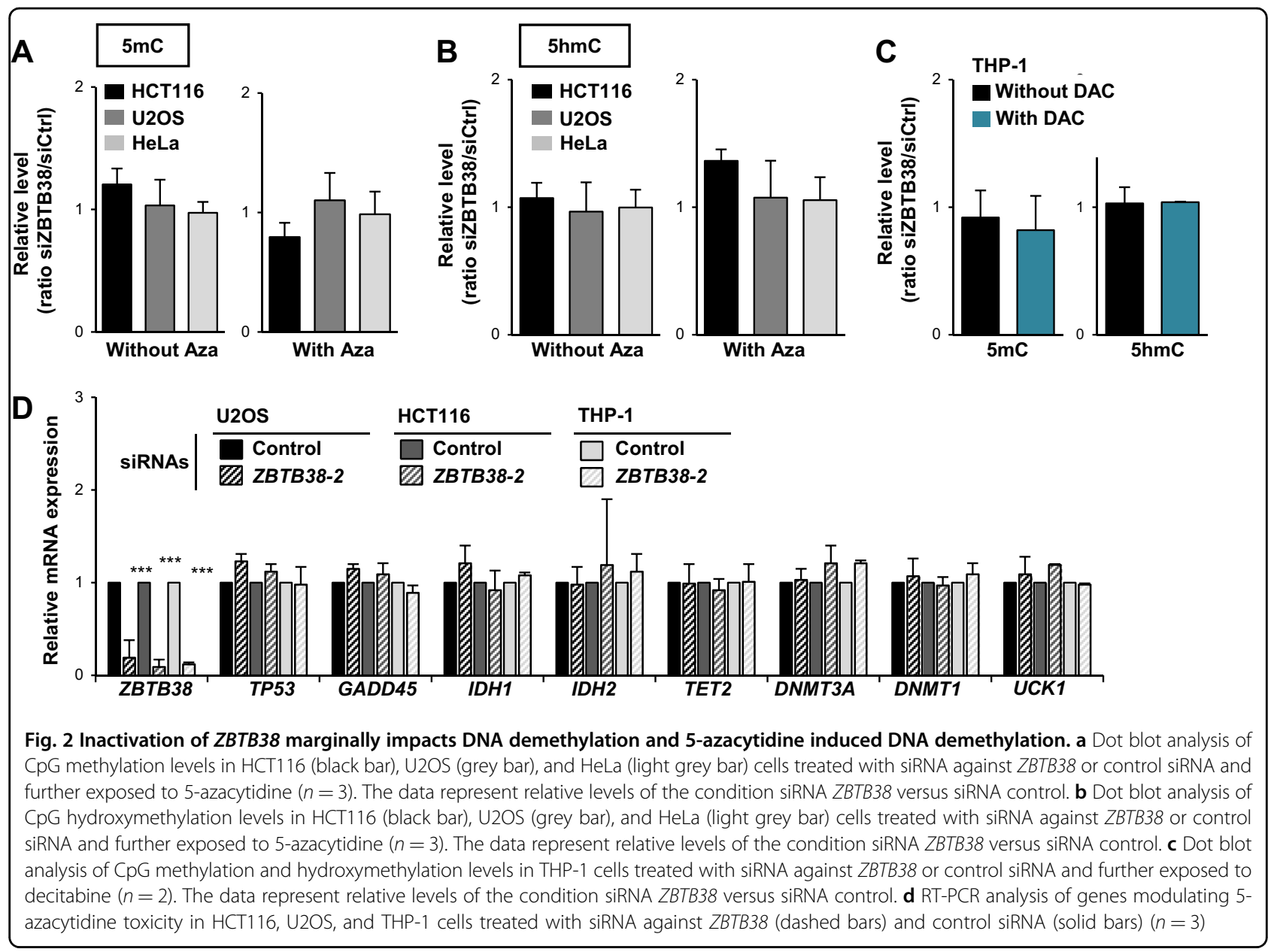

in the establishment and/or maintenance of DNA methylation.

\section{Depletion of ZBTB38 using specific anti-sense RNA molecules enhances 5-azacytidine cytotoxicity in cancer cells}

We tested whether ZBTB38 inactivation would modulate the cytotoxicity of 5-azacytidine in cancer cells. Cells were transfected with siRNAs molecules directed against ZBTB38 and $48 \mathrm{~h}$ later 5-azacytidine (concentration ranging from 1 to $10 \mu \mathrm{M}$ ) was added to the culture media for $48 \mathrm{~h}$. Fresh media (without drug) was then replaced every 3 days for two weeks and cell colony counting was performed at that stage. We observed that depletion of ZBTB38 with specific siRNAs causes a significant inhibition in the formation of HeLa colonies at different concentrations of 5-azacitidine (Fig. 3a, b). This effect was confirmed in other cancer cell types, notably HCT116, HCT116 TP53 ${ }^{-1-}$, HCT116 DKO, U2OS, and DU145 (Fig. 3c). We also monitored the proliferation of leukemia cells: K562, THP-1, and MOLM-14. Cells were transfected with siRNA molecules and $48 \mathrm{~h}$ later exposed to 5- azacytidine for $48 \mathrm{~h}$. Live cell counting was performed at that stage (Fig. 3d). Again the depletion of ZBTB38 enhanced the deleterious effects of 5 -azacytidine and the amplitude of the cytotoxicity caused by ZBTB38 depletion was variable among the different cell types.

We then extended the analysis to decitabine and zebularine on a panel of cell types. On its own, decitabine inhibits cell growth in a concentration dependent manner (Supplementary Fig. S3A). Depletion of ZBTB38 further enhanced the cytotoxicity of decitabine in all tested cell types (Supplementary Fig. S3A). Zebularine barely impact cell growth even at the highest concentration we tested (Supplementary Fig. S3B). However, ZBTB38 silencing significantly inhibits colony formation in the presence of zebularine, in the two cell types tested (Supplementary Fig. S3B). Altogether, our results demonstrate that depletion of $Z B T B 38$ enhances the cytotoxicity of several DNMT inhibitors in various cancer cells.

In order to further demonstrate that the depletion of ZBTB38 enhances the cytotoxicity to DNMTi, we compared the colony formation capacity of HeLa and U2OS cells when ZBTB38 depletion and decitabine exposure are 


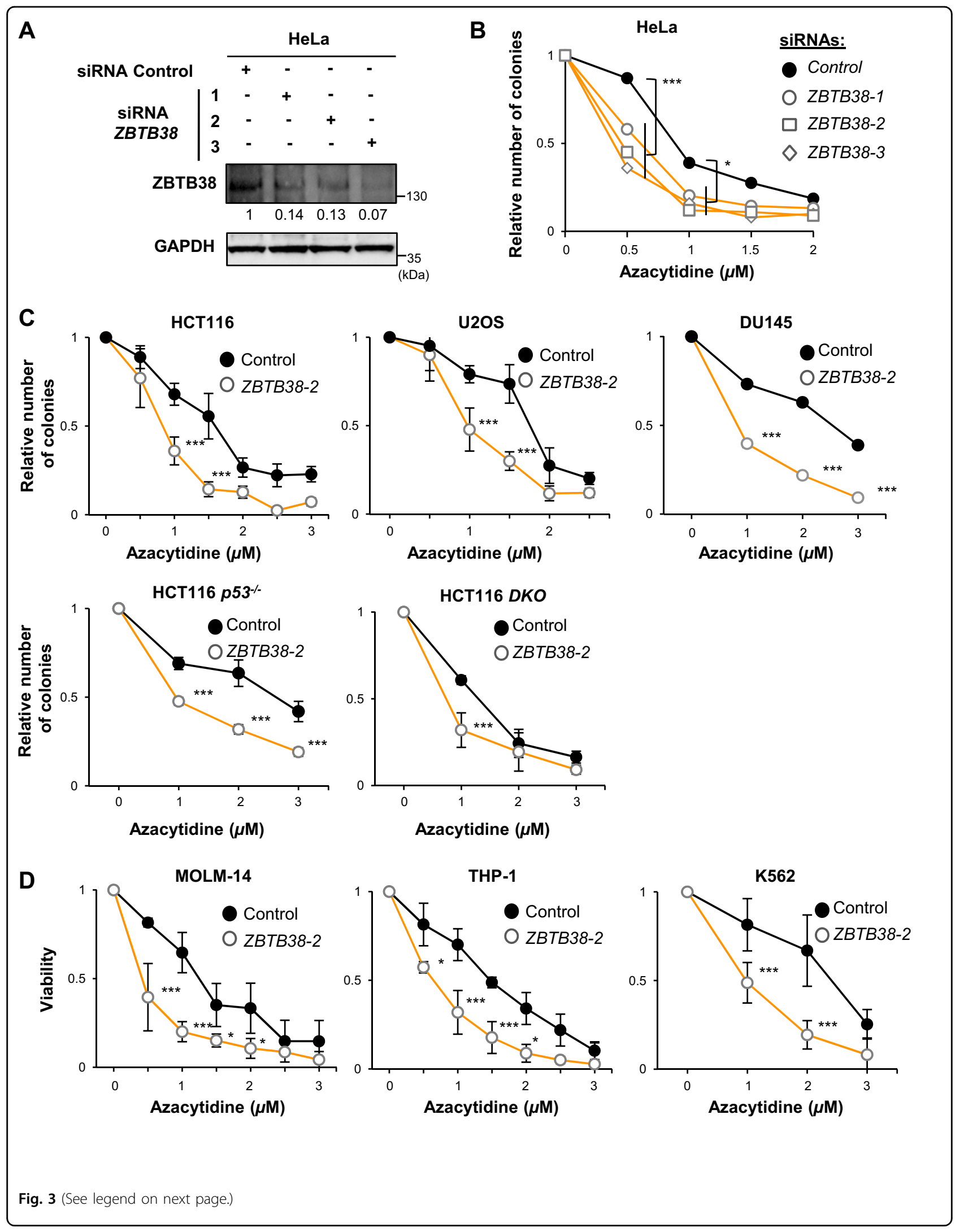


(see figure on previous page)

Fig. 3 Inactivation of ZBTB38 by RNA interference enhances the cytotoxicity of 5-azacytidine in a variety of cancer cells. a Western blot analysis of ZBTB38 and GAPDH expression in cells treated with three different anti-sense RNA directed against ZBTB38 and a control anti-sense RNA. Relative expression level of ZBTB38 is indicated under the blots with the control condition set up as 1. $\mathbf{b}$ Depletion of ZBTB38 enhances the cytotoxicity of 5 -azacytidine in colony formation assays of HeLa cells $(n=3)$. Colony were counted 15 days post-azacytidine exposure and normalized to the condition without 5 -azacytidine, artificially set up as $1 .{ }^{* *} P<0.001 ;{ }^{*} P<0.05$. c Depletion of ZBTB38 enhances the cytotoxicity of 5 -azacytidine in colony forming assays of HCT116, U2OS, DU145, HCT116 $p 53^{--1-}$ and HCT116 DKO cells $(n=4){ }^{* * *} P<0.005$. d Depletion of ZBTB38 enhances the cytotoxicity of 5 -azacytidine in leukemia K562, THP-1, and MOLM-14 cells $(n=3)$. Cell viability was analyzed 2 days post-azacytidine exposure by scoring trypan blue-positive cells and reported as the number of viable cells normalized to the condition without 5-azacytidine, artificially set up as 1. ${ }^{* *} P<0.001 ; * P<0.05$

simultaneous and when ZBTB38 depletion is performed after decitabine exposure. We observed that the number of colonies was similar between control and ZBTB38depleted cells when ZBTB38 depletion is performed after decitabine exposure (Supplementary Fig. S3C-E). This result indicates there is no synergistic effect when cells are exposed to DNMTi prior to ZBTB38 depletion, supporting our hypothesis that DNMTi cytotoxic effects should be at least partially mediated by $Z B T B 38$.

\section{Transient depletion of ZBTB38 or USP9X combined with decitabine exposure causes a long-term arrest of leukemia cell proliferation}

We investigated the kinetics of cell proliferation when ZBTB38 depletion is combined with decitabine or azacytidine exposure in leukemia cells. We treated K562, THP-1, and MOLM-14 cells with decitabine $(1 \mu \mathrm{M}$, a dose achieved in human plasma ${ }^{3}$ for $24 \mathrm{~h}$. After this period, the cells were washed with fresh media (without decitabine) and proliferation was monitored for 6 days. Under normal condition of cell culture, inactivation of ZBTB38 by RNA interference barely impacted the proliferation of K562 and THP-1 cells, while it stimulated the proliferation of MOLM-14 cells (Fig. 4a-c). Treatment with decitabine causes a strong inhibition of cell proliferation but cells eventually tend to regain cell proliferative abilities on day 4 or 5 post-decitabine treatment depending on the cell type (Fig. 4a-c). On the contrary, ZBTB38-depleted cells were not capable to regain proliferative abilities until the end of the experiment (Fig. 4a-c). We thus concluded that the transient depletion of ZBTB38 at the time of decitabine exposure negatively impact leukemia cell proliferation even after the return to the normal culture conditions.

We next tested the consequences of ZBTB38 depletion and azacytidine treatment on cell proliferation. We observed a stronger inhibition of cell proliferation when azacytidine treatment is combined with $Z B T B 38$ silencing compared to azacytidine alone (Fig. $4 \mathrm{~d}$ ). The defect in cell proliferation is even more pronounced than upon decitabine treatment and it is recapitulated with two different siRNAs against ZBTB38 (Fig. 4d). These observations indicate that the transient silencing of $Z B T B 38$ at the time of DNMTi treatment negatively affects the proliferation of leukemia cells.

We further supported this conclusion using two additional approaches. We tested the consequences of USP9X depletion and decitabine exposure on cell proliferation. We transfected THP-1 and MOLM-14 cells with two different siRNAs against USP9X and treated the cells with decitabine $(1 \mu \mathrm{M})$ for $24 \mathrm{~h}$. After this period, the cells were washed with fresh media (without decitabine) and proliferation was monitored for 7 days. Without DNMTi, inactivation of $U S P 9 X$ by RNA interference barely impacted the proliferation of MOLM-14 cells, while it stimulated the proliferation of THP-1 cells (Supplementary Fig. S4A-B). USP9X depleted cells were barely able to proliferate in presence of DAC compared to control cells that grow very slowly (Fig. 4e and Supplementary Figure S4A-B). The expression of ZBTB38 was very low in both cell lines silenced for USP9X compared to control, further supporting that low ZBTB38 protein level enhances DNMTi toxicity (Supplementary Fig. 4A-B). In a second experiment we utilized a different protocol of DAC exposure. We used lower levels of DAC $(0.1 \mu \mathrm{M})$ that was renewed every day for 5 days. In this experimental set up, we also observed that cells depleted of ZBTB38 or USP9X proliferate slowly than control siRNA transfected cells (Supplementary Fig. S4C). This suggests that depletion of ZBTB38 or its deubiquitinase USP9X enhances DAC toxicity in THP1 and MOLM-14 cells independently of DAC exposure protocol.

\section{Depletion of ZBTB38 causes a significant increase in cell death upon DNMTi exposure in solid and hematologic cancer cells}

We conducted an analysis of the cell cycle, cell death, and autophagy by flow cytometry in HeLa and THP-1 cells transfected with or without ZBTB38 siRNAs and further exposed or not to decitabine. We could not find evidence of significant changes in autophagy and cell cycle progression in cells combining ZBTB38 depletion and DNMTi exposure compared to DNMTi alone (Supplementary Fig. S5A-E). We however observed a modest increased in cell death in cells combining ZBTB38 depletion and decitabine exposure. This was mostly 


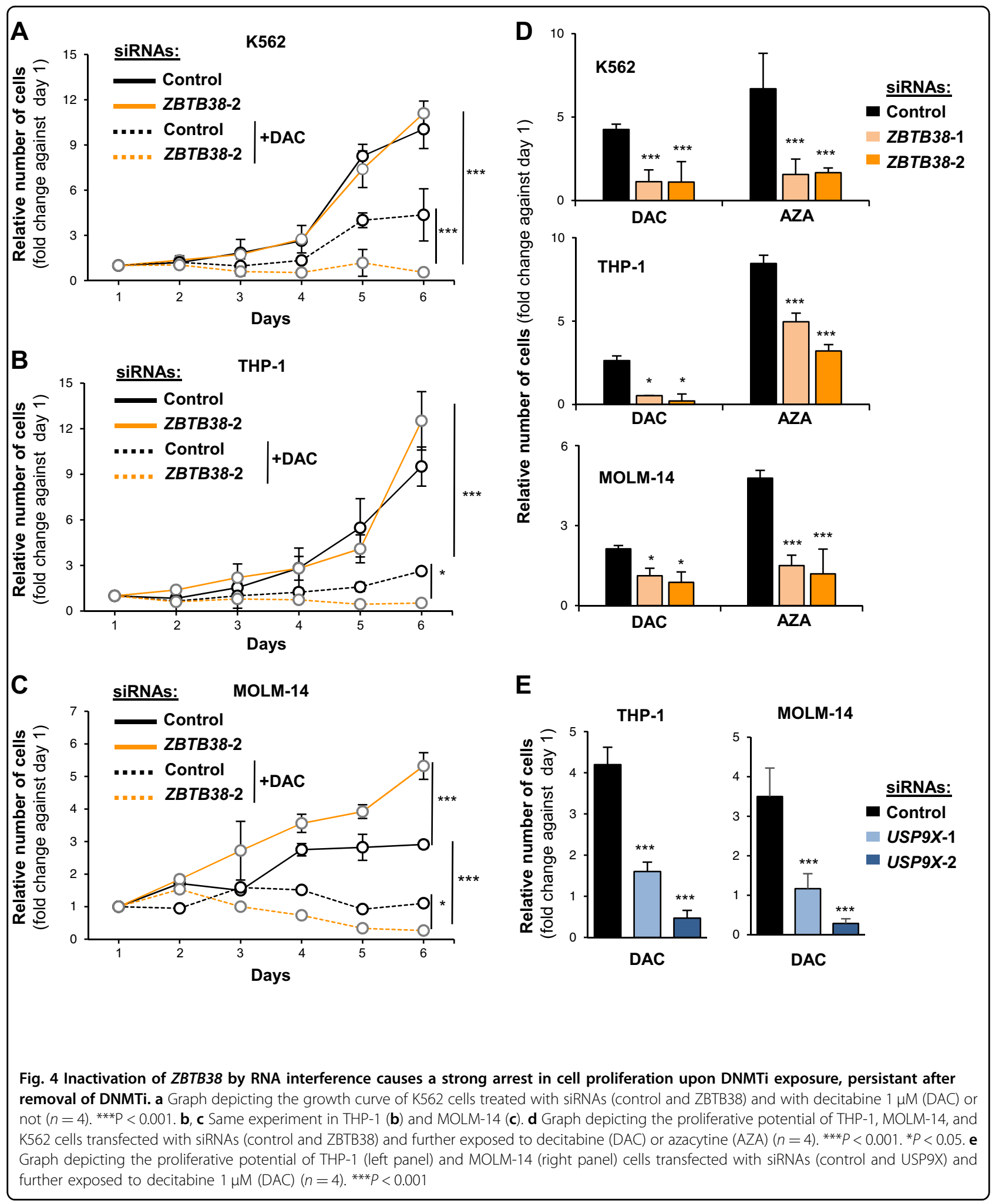


A

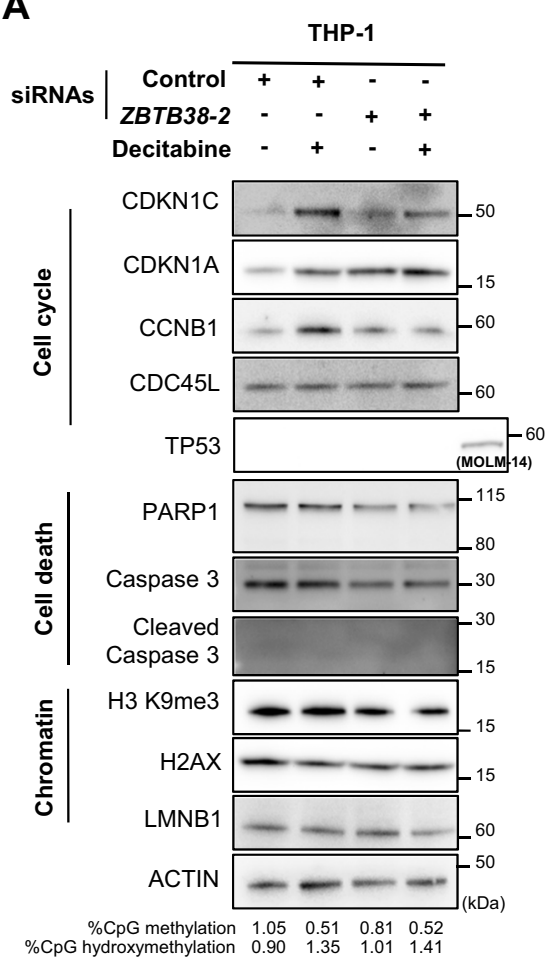

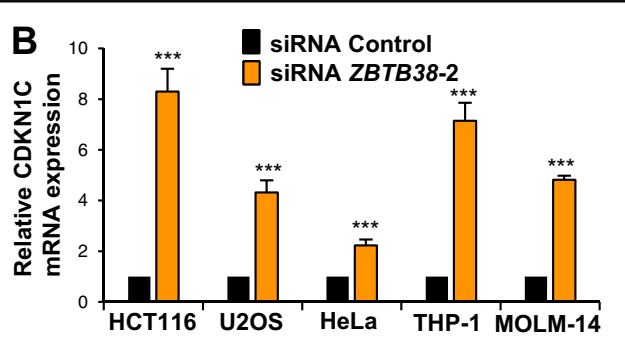

C
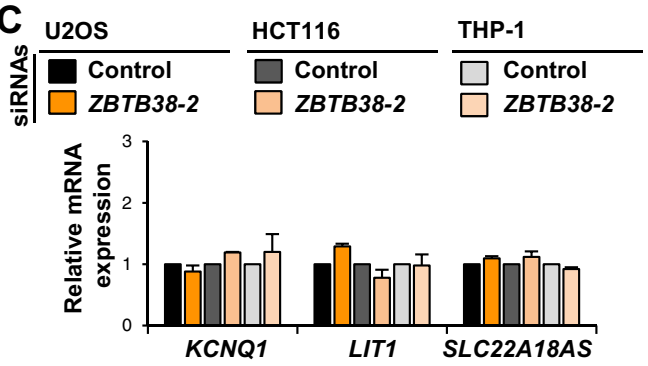

D

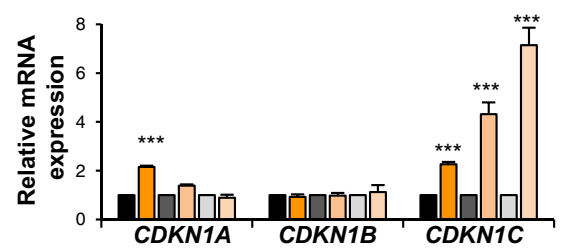

E siRna Control SIRNA USP9X-1

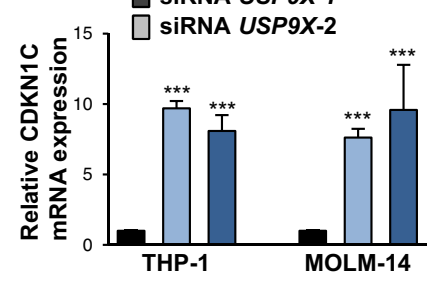

F

U2OS $\frac{\text { HeLa }}{++-} \frac{\text { HCT116 }}{++-}$ SIRNA ZBTB38 Azacytidine CDKN1C CDKN1A GAPDH
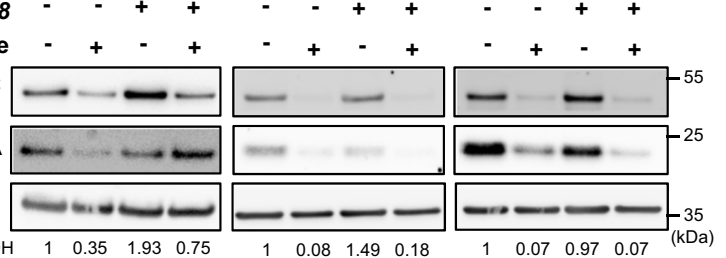

G
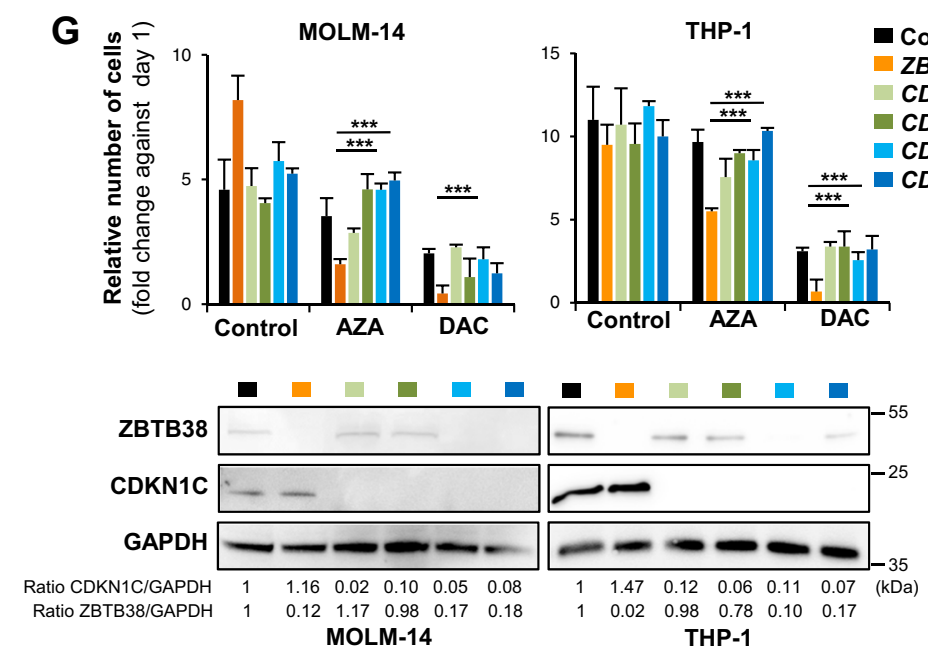

Fig. 5 (See legend on next page.) 
(see figure on previous page)

Fig. 5 Depletion of ZBTB38 induces CDKN1C up-regulation at the mRNA level in solid and hematologic cancers. a Western blot analysis of cell death, cell cycle, and DNA replication factors expression in THP-1 cells treated with siRNAs (control and ZBTB38) and with decitabine (1 $\mu \mathrm{M}$ for $24 \mathrm{~h}$ ) or not. The experiment was performed on day 1. Levels of $5 \mathrm{mC}$ and $5 \mathrm{hmC}$ evaluated by dot blot are indicated. TP53 expression in MOLM-14 cells is also presented as control. b Analysis by RT-qPCR of CDKN1C expression in U2OS, HCT116, HeLa, THP-1, and MOLM-14 cells treated with an siRNA against ZBTB38 and control siRNA $(n=3)$. c RT-qPCR analysis of the expression of genes located in the vicinity of CDKN1C at the ICR1 locus in HeLa, HCT116, and THP-1 cells $(n=3)$. d Analysis by RT-qPCR of CDKN1A, CDKN1B, and CDKN1C expression levels in HeLa, HCT116, and THP-1 cells treated with an siRNA against ZBTB38 and control siRNA $(n=3)$. e Analysis of CDKN1C expression by RT-qPCR in THP-1 and MOLM-14 cells transfected with siRNA against USP9X or control siRNA $(n=3)$. f Western blot analysis of CDKN1C protein expression in U2OS, HeLa, and HCT116 cells challenged with 5-azacytidine $(4 \mu \mathrm{M}$ for $24 \mathrm{~h})$ and further treated with siRNAs against ZBTB38. $\mathbf{g}$ Depletion of CDKN1C rescues the cytotoxicity caused by DNMTi and ZBTB38 silencing in THP-1 and MOLM-14 cells $(n=3)$. Cell viability was analyzed 2 days post-DNMTi exposure (azacytidine: $1 \mu \mathrm{M}$ for $24 \mathrm{~h}$ and decitabine: $2 \mu \mathrm{M}$ for $24 \mathrm{~h}$ ) by scoring trypan blue-positive cells and by reporting the ratio between the number of viable cells at Day 2 compared to Day $0 .{ }^{* *} P<0.05$. A representative western blot of CDKN1C and ZBTB38 protein expression in each condition is shown for one of the three replicate

noticeable by an increase in necrotic cells (positive for propidium iodide and negative for annexin $\mathrm{V}$ ) and by the presence of cell debris (Supplementary Fig. S5F-H).

\section{Depletion of ZBTB38 increases the expression of CDKN1C at mRNA level}

We analyzed the expression of several markers of cell death and cell cycle arrest by western blot in cellular extracts prepared from THP-1 cells transfected with or without ZBTB38 siRNAs and further exposed or not to decitabine. We investigated the proteolytic cleavage of PARP-1 and Caspase3. Consistent with FACS analyses we could not observe increased cleavage of PARP-1 and Caspase3 in cells combining ZBTB38 depletion and decitabine compared to all other experimental conditions (Fig. 5a). Among all the other factors investigated, we observed an alteration in CDKN1C (also known as p57 and KIP2), CDKN1A (p21 or WAF1), and CCNB1 (Cyclin B1) protein expression levels (Fig. 5a). CDKN1C and CCNB1 expression levels were higher in ZBTB38 depleted cells compared to control cells under normal culture conditions (Fig. 5a). Differing from untreated conditions, CDKN1C and CCNB1 protein expression levels were lower in cells combining ZBTB38 depletion and decitabine exposure compared to decitabine alone. CDKN1A/ p21 expression was higher in cells depleted of ZBTB38, exposed to decitabine and combining both treatments compared to control (Fig. 5a). These findings indicated that specific cell pathways might be altered when ZBTB38 depletion is combined with DNMTi exposure.

We further focused our study on CDKN1C and ZBTB38 for three main reasons. First, $C D K N 1 C$ is known for its role in the regulation of cell growth, cellular senescence, and MDS development ${ }^{36,37}$. Second, CDKN1C is an indirect transcriptional target of azacytidine and decitabine in multiple cancer cells ${ }^{1,3,22}$. Third, ZBTB38 paralogs, ZBTB4 and ZBTB33, regulate the expression of CDKN1 family genes in cancer cells ${ }^{38-41}$.
We first investigated the expression of CDKN1C mRNA in different cell types treated with siRNAs against ZBTB38. By RT-qPCR, we observed that depletion of $Z B T B 38$ induces an up-regulation of CDKN1C mRNA expression in all the cell types tested (Fig. $5 \mathrm{~b}$ ). CDKN1C is located in a region of chromosome 11 containing many imprinted genes and long non coding RNAs, with complex direct and indirect transcriptional regulation ${ }^{42}$. We studied the expression of CDKN1C neighboring genes in HeLa and THP-1 cells silenced for ZBTB38. ZBTB38 knock-down did not change KCNQ1, SLC22A18AS and the long non coding RNA LIT1 (or KCNQ1OT) expression levels compared to control cells (Fig. 5c). We also observed that CDKN1A and CDKN1B mRNA expression levels were marginally affected by the depletion of ZBTB38 (Fig. 5d). We also investigated CDKN1C mRNA expression in THP1 and MOLM-14 cells treated transfected with two different siRNAs against USP9X. We observed the up-regulation of CDKN1C mRNA expression in cells depleted of USP9X compared to control (Fig. 5e).

We then investigated the expression of CDKN1C in different cancer cells by western blot (Fig. 5f). Depletion of ZBTB38 causes CDKN1C protein up-regulation in U2OS and HCT116 cells to different extent, and no significant up-regulation in HeLa cells (Fig. 5f). When cells were further challenged with 5-azacytidine we observed a down-regulation of CDKN1C at protein level in U2OS and HCT116 but the amount of CDKN1C remained higher in the siRNA ZBTB38 condition compare to control cells (Fig. 5f). Depletion of ZBTB38 causes an upregulation of CDKN1C mRNA and protein in U2OS, HCT116, and THP-1 cells under normal and DNMTi condition. The lack of correlation between CDKN1C mRNA and protein expression levels is not unprecedented $^{43-45}$ and suggests that ZBTB38 depletion might alter both the transcriptional and post-transcriptional regulation of CDKN1C. 


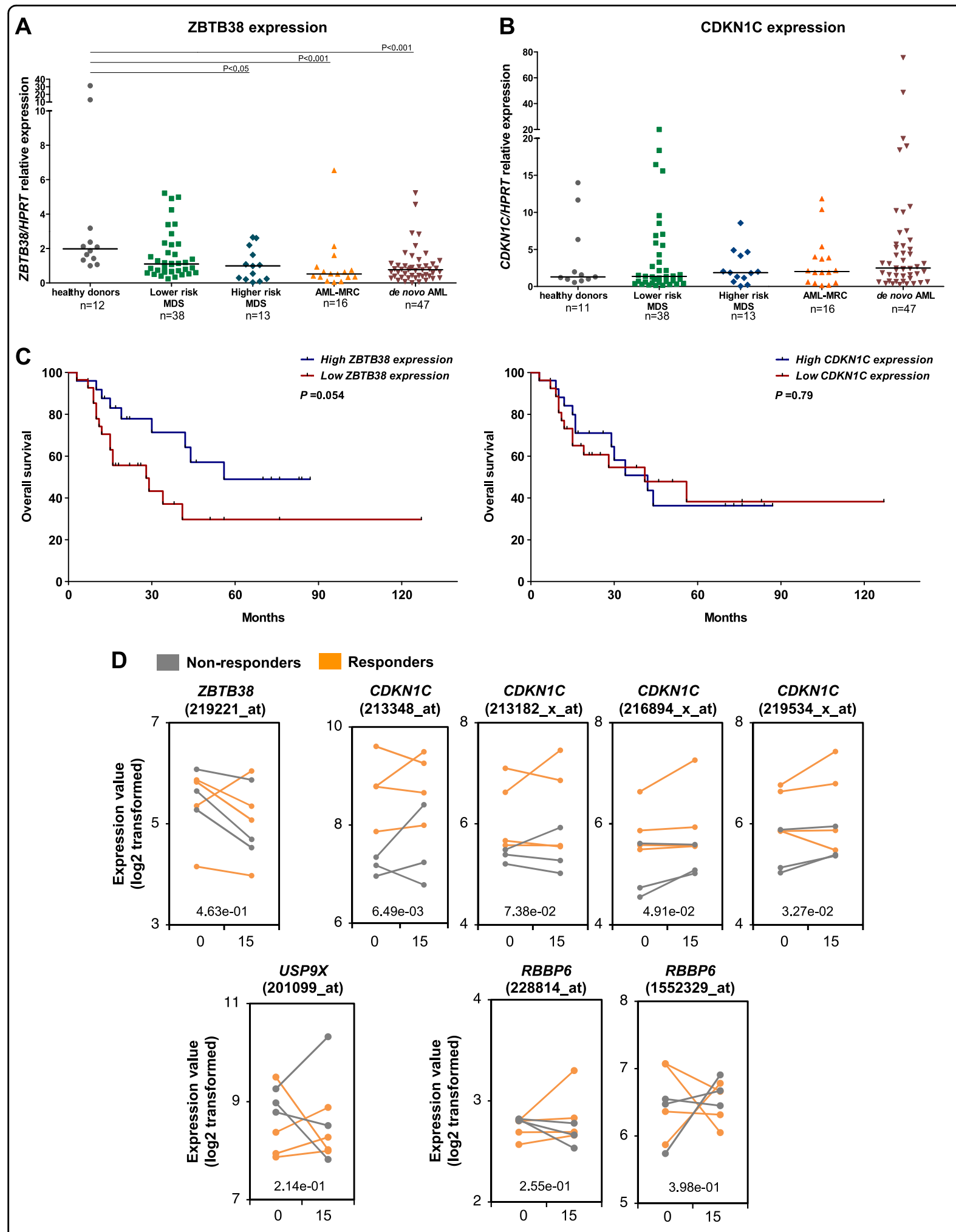

Fig. 6 (See legend on next page.) 
(see figure on previous page)

Fig. 6 High expression of CDKN1C mRNA in the bone marrow of patients with MDS coincides with a better response to 5-azacytidine plus entinostat. a RT-qPCR analysis of ZBTB38 and $\mathbf{b}$ CDKN1C mRNA expression in bone marrow samples from patients with MDS, AML with

myelodysplasia-related changes (AML-MRC) and de novo AML. MDS patients were grouped into lower and higher-risk categories according to the Revised International Prognostic Scoring System. c Kaplan-Meier plots of overall survival in MDS patients classed according to low and high ZBTB38 (left panel) or CDKN1C (right panel) mRNA expression levels. $\mathbf{d}$ Expression of ZBTB38, RBBP6, USP9X, and CDKN1C in samples from patients with MDS before azacytidine and entinostat treatment (D0) and 15 days later (D15). Responders to the therapy are highlighted in orange; non responder are presented in grey (data from ref. ${ }^{1}$ ). Significant difference between responder and non-responder at Day 0 is indicated by a $P$-value in the graph

We tested whether CDKN1C silencing alter the response to azacytidine or decitabine in MOLM-14 and THP-1 cells. Following transfection of cells with siRNAs against CDKN1C, we exposed the cells to DNMTi and monitored cell survival over 2 days. Depletion of CDKN1C has a minor, if any, impact on the viability of THP-1 and MOLM-14 cells in absence or in presence of azacytidine and decitabine (Fig. 5g). However, cells codepleted of ZBTB38 + CDKN1C showed a very similar viability as control cells in absence or in presence of DNMTi (Fig. 5g). These experiments suggest that CDKN1C depletion prevents cell defects caused by ZBTB38 depletion and that CDKN1C up-regulation contributes to ZBTB38 toxicity upon DNMTi treatment.

\section{High levels of CDKN1C mRNA expression are associated with a better response to a combined 5 -azacytidine and entinostat treatment in MDS patients}

We investigated $Z B T B 38$ and CDKN1C expression in bone marrow samples from MDS and AML patients by quantitative PCR (Fig. 6a, b). Data is showed as median [max-min]. ZBTB38 expression was decreased in AML with myelodysplasia-related changes (AML-MRC) $(0.52$ [6.54-0.04]) and in de novo AML (0.77 [5.23-0.01]), compared to healthy donors (1.98 [31.47-1.00]), both $P<$ 0.001 (Fig. 6a). When MDS patients were stratified according to Revised International Prognostic Scoring System (IPSS-R) in lower risk (very low/low/intermediate) and higher risk (high/very high), ZBTB38 was significantly reduced in higher-risk MDS (0.99 [2.65-0.04]) in comparison to healthy donors, $P<0.05$ (Fig. 6a). These results are in agreement with previously published microarray datasets from patients with MDS and $\mathrm{AML}^{46}$, that showed that ZBTB38 mRNA is down-regulated in AML compared to control individuals (Supplementary Fig. S6A). CDKN1C mRNA expression did not significantly differ between patients with AML or MDS and healthy donors (Fig. 6b). It is possible that the expression of ZBTB38 is altered during cell differentiation and that its reduction in AML reflects the increased number of blasts. Nevertheless, our data suggest that the decrease in ZBTB38 expression is a possible marker of AML and high-risk MDS.

We further accessed the association between ZBTB38 or CDKN1C mRNAs levels and the overall survival and event free survival of MDS patients in our cohort (most of them were not receiving hypomethylating agents). With an average follow-up of 30 months, we found that ZBTB38 and CDKN1C mRNA levels do not independently predict overall survival or event free survival in MDS (Supplementary Table 1). IPSS-R was an independent prognostic factor for worse overall survival (HR 3.82 [95\% CI, 1.5-9.4], $P=0.004)$, validating our cohort (Supplementary Table 1). Kaplan-Meier analysis indicated a poorer survival of MDS patients with lower ZBTB38 expression (below the median) in comparison with higher ZBTB38 (above the median), although not statistically significant $(P=0.054)$ (Fig. 6c). Overall, ZBTB38 mRNA expression is lower in higher-risk MDS patients and AML patient compared to healthy donors but does not predict overall survival and event free survival in MDS patients.

We then directly investigated the relationship between RBBP6, USP9X, ZBTB38, and CDKN1C expression and the clinical response to azacytidine in cancer patients. We retrieved the data reported for seven patients with MDS treated with 5-azacytidine plus entinostat (a potent HDACi $)^{1}$. Three patients did not respond to chemotherapy, three patients had a partial clinical response and one patient presented a complete response. For each patient we have access to gene expression in the bone marrow prior to and 15 days after chemotherapy began. We observed no significant differences in the level of $Z B T B 38$, $R B B P 6$, and USP9X mRNA expression in samples from responder and non-responder patients at day 0 and at day 15 (Fig. 6d). In addition, we did not observe significant changes in the ZBTB38, RBBP6, and USP9X mRNA levels between day 0 and day 15 of treatment (Fig. 6d). On the contrary, we observed that CDKN1C mRNA expression was significantly higher in samples from responders compared to samples from non-responders at screening, day 0 (Fig. 6d). No clear difference is observed if the analysis is reproduced at day 15 post treatment (Fig. 6d). We propose that $C D K N 1 C$ mRNA expression in MDS might be used to identify DNMTi responders but is not well suited to monitor drug response. Nonetheless, the small sample size of this study precludes a definitive conclusion on the predictive power of CDKN1C mRNA expression. Of note, we still observed the same trend 
when we extended the analysis to AML and CMML patients (4 extra-individuals) (Supplementary Figure S6B).

\section{Discussion}

ZBTB38 protein abundance is decreased by DNMTi

Cancer cells generally present global and local alteration in the pattern of DNA methylation ${ }^{1,3,18,23}$. In that framework, it has been proposed that DNMTi and other modulators of DNA methylation may have beneficiary effect in cancer treatments. In this study we found that 5 azacytidine and its derivatives cause the down regulation of ZBTB38 protein level without significantly affecting ZBTB38 mRNA expression or the expression of its paralogs ZBTB4 and ZBTB33/KAISO.

Decitabine, azacytidine, and zebularine are viewed as different types of DNMT inhibitors due to their structure, their mode of incorporation into the DNA as well as their metabolic fate ${ }^{2,3,22,23}$. These observations indicate that ZBTB38 degradation is most likely due to an indirect consequence of DNMTi cytotoxicity. We showed that ZBTB38 expression is not perturbed by treatments with siRNAs against DNMT1 or in HCT116 DKO cells that present different levels of DNA methylation loss; and that caffeine treatments dampens ZBTB38 downregulation upon azacytidine treatment, consistent with the involvment of a damage-induced signalisation in ZBTB38 regulation.

We also provide evidence that ZBTB38 polyubiquitination is involved in the control of ZBTB38 stability upon DNMTi treatment. While deubiquitinase USP9X is not mys-regulated upon azacytidine treatment in HeLa cells, E3 ubiquitin ligase RBBP6 is upregulated in DNMTi treated cells ${ }^{31}$. RBBP6 is involved in the maturation of messenger RNAs (mRNA) and controls the stability of a number of mRNAs in human cells ${ }^{47}$. This function of RBBP6 implicates structural domains present within the $\mathrm{N}$-terminal portion of the protein and shared between different RBBP6 isoforms ${ }^{47}$. It is thus possible that DNMTi, by causing mRNA and DNA adducts and/or demethylation alters the expression, stability and/or function of RBBP6 isoforms and, in turn, contribute to the down-regulation of ZBTB38 protein expression.

\section{Implication of ZBTB38 in the toxicity of 5-azacytidine and its derivatives}

Many models have been proposed to explain the toxicity of 5-azacytidine in cancer cells. Here, we demonstrate that transient inhibition of ZBTB38 expression by siRNAs enhances the toxicity of 5-azacytidine, and its derivatives, in different cancer types. The consequences are dependent on the cell type owing to the presence of additional alterations affecting the sensibility to DNMTi (for example TP53).
Our investigations indicate that depletion of ZBTB38 does not enhance the demethylating activity of 5azacytidine or the conversion of 5-methyl-cytosine into 5-hydroxy-methyl-cytosine. Consistent with this observation, we found that the genes encoding DNMT1, TET2, or $I D H 1 / 2$ enzymes were not regulated by $Z B T B 38$.

We identified a recurrent up-regulation of CDKN1C expression at the mRNA level in different cancer cell types silenced for ZBTB38 or its deubiquitinase USP9X. However, when we studied the expression of $C D K N 1 C$ at the protein level, we observed either a down-regulation or an upregulation in ZBTB38 silenced cells. A likely explanation is that $Z B T B 38$ down-regulation disrupts $C D K N 1 C$ regulation transcriptionally and posttranslationally in presence of DNMTi. The analysis of ZBTB38 genomic targets by chromatin immunoprecipitation does not identify binding sites for ZBTB38 in the ICR2 region nor at the CDKN1C promoter in HeLa cells (C.M., P.A.D. and B.M., unpublished data). The regulation is thus likely to be indirect or the recruitment of ZBTB38 highly dynamic at the promoter of $C D K N 1 C$.

The $11 \mathrm{p} 15.5$ region containing CDKN1C is associated with growth disorders such as the Silver-Russell syndrome and the Beckwith-Wiedemann syndrome ${ }^{42,48}$. Intriguingly many polymorphisms in the ZBTB38 locus are linked to the regulation of human height and to idiopathic growth syndrome and some of these polymorphisms affect $Z B T B 38$ expression level ${ }^{49,50}$. It is thus tempting to speculate that $Z B T B 38$ might regulate the expression of $C D K N 1 C$ in pathological but also physiological context including during embryonic development. Further studies will be required to clarify the direct and indirect regulation of $C D K N 1 C$ expression by ZBTB38 in normal and cancer cells. It is also likely that additional targets and pathways are mys-regulated upon ZBTB38 depletion and that they may also contribute to ZBTB38 effects, explaining variable effects on CDKN1C protein levels and DNMTi toxicity in different cell types.

\section{Can ZBTB38 inactivation further enhance the efficacy of DNMTi inhibitors in clinic?}

Prediction of cancer drug response is an important challenge ${ }^{8,51,52}$. Markers able to predict the response to DNMTs inhibitors and individuals most likely to benefit from the therapy have been largely unsuccessful and not yet validated in clinic $^{1,9,21,51-53}$.

To test the possibility that USP9X, RBBP6, ZBTB38, and/or CDKN1C could be predictive markers, we retrieved the clinical and gene expression data from a previously published cohort of patients with MDS showing effective and tolerable effects of 5 -azacytidine ${ }^{1}$. Using this information on seven patients, we observed a correlation between a high level of $C D K N 1 C$ mRNA expression before treatment and the clinical response to 5- 
azacytidine in patients with MDS. In haematopoietic cells derived from MDS and AML patients the CDKN1C promoter is mostly unmethylated ${ }^{1}$. The baseline level of CDKN1C mRNA expression prior treatment might thus help the identification of patients could benefit from a chemotherapy based on a combination of DNMTi and HDACi. In addition, enhancing CDKN1C mRNA basal expression by disrupting ZBTB38 or USP9X might help further enhance the response to DNMTi in clinic or define new chemotherapeutic cocktails.

The analysis of our own cohort of patients, that for most of them were not receiving hypomethylating agents, indicate that ZBTB38 and CDKN1C mRNA levels do not predict overall survival in these patients with MDS. Previous works indicate that CDKN1C protein expression scored by immunohistochemistry (but not mRNA expression) prior to treatment help anticipate the clinical response of patients with MDS to conventional chemotherapy ${ }^{37}$. These data suggest that monitoring both CDKN1C mRNA and protein expression levels in patients might help classify potential responders to 5-azacytidine treatment or conventional care therapy. Our study grants the analysis of a larger number of patients with MDS/ AML under hypomethylating therapy or conventional care regimens to further support the observation that CDKN1C mRNA might help predict 5-azacytidine efficacy. Increasing the number of patients will also allow the consideration of genomic abnormalities and clinical features in patients that could mask or unravel CDKN1C predictive value, including the status of tumor suppressor TP53 and DNMTs. The status of CDKN1C expression could also be tested in other cancers as 5 -azacytidine plus entinostat regimen has been successfully tested on patients with AML arising after chemotherapy or radiation therapy, breast cancer and colorectal cancer ${ }^{5,7}$.

Our work provides a better understanding of 5azacytidine action in cancer cells and it might help improve current predictive strategies for determining good outcome and survival upon 5-azacytidine-based therapy in cancer.

\section{Materials and methods}

\section{Cell culture conditions}

The cells were grown in a humidified atmosphere of 5\% $\mathrm{CO}_{2}$ at $37^{\circ} \mathrm{C}$ and the media changed every 2 days. A detailed description of culture condition and media composition is provided in the supplementary section.

\section{Chemicals and siRNAs}

5-Azacytidine (Sigma-Aldrich; A1287), 5-Aza-2'-Deoxycytidine/Decitabine (Sigma-Aldrich; A3656) and Pyrimidin-2-one Beta-Ribofuranoside/Zebularine (SigmaAldrich; Z4775) were freshly resuspended in DMSO at the concentration recommended by supplier. The suspension was further diluted in the culture media for the experiment to final working concentration of up to $10 \mu \mathrm{M}$. Cells were treated with media containing 5-azacytidine (or derivatives) replaced every $24 \mathrm{~h}$ to ensure proper action of the drug(s) for long-term expositions. Proteasome inhibitor MG132 was purchased from Merck Millipore (InSolution ${ }^{\mathrm{TM}}$ MG132) and it was used at final concentration of $10 \mu \mathrm{M}$ for $4 \mathrm{~h}$. Caffeine was purchased from Sigma-Aldrich (C0750) and it was used at final concentration of $1 \mathrm{mM}$ for $24 \mathrm{~h}$.

Control scrambled siRNAs and siRNAs directed against ZBTB38, USP9X, p53, CDKN1C and DNMT1 were purchased from Thermo Fisher Scientific and some were validated in previous studies ${ }^{31,32}$. siRNAs were delivered in adherent cells using Lipofectamine 3000 following protocols provided by the manufacturer (Thermo Fisher Scientific). Cells in suspension (K562, MOLM-14 and THP-1) were electroporated using the Neon transfection system (Thermo Fisher Scientific) using the kit MPK10096 and the following parameters: pulse voltage at $1450 \mathrm{~V}$, pulse width 10 milliseconds and a total of 3 sequential pulses.

\section{Colony forming assays}

A fixed number of adherent cells (200 cells) were seeded in 6-well plates. Typically, each plate is prepared in triplicate to control plating efficiency and drug efficiency. After $24 \mathrm{~h}$ in the incubator the cells were exposed to different concentration of 5 -azacytidine (or derivatives) for $48 \mathrm{~h}$. The cells were then incubated in fresh media for up to 15 days (depending on the cell type). The cells were fixed, under a fume hood, with formaldehyde, stained with crystal violet and extensively washed with distilled water. The number of colonies per well was then manually counted and also access using the Image J software on scanned images of the plates. Concordant results were further considered, and the ratio between the number of colonies in the treated condition normalized to the number of colonies in the control condition reported in graphs.

\section{Cell proliferation assay with suspension cells}

K562, MOLM-14 and THP-1 cells were seeded in 6-well plate and treated with adequate concentrations of DNMTi. At the different time points the number of cells per well was manually monitored using a KOVA slide with counting grid. Each experiment was technically replicated and we performed at least three independent biological replicate. The condition without DNMTi was used as a control and all data in each experiment were expressed relative to its value (set up to 1 ). In the case of proliferation curve analysis the data are expressed relative to the number of cells at day 0 (day of DNMTi removal) in each condition. 


\section{GEO datasets and bioinformatics analysis}

The different datasets analyzed in this study were retrieved from the NCBI gene expression omnibus portal from previously published work and are detailed in the supplementary section.

Correlation between gene expression levels in multiple conditions was accessed by Pearson's coefficient analysis and presented as the square of the Pearson's coefficient in graphs. Comparison of mean ranks was accessed by Mann-Whitney $U$-test and the $p$-value reported.

\section{DNA methylation analysis}

Global and site-specific analysis of DNA methylation were conducted using the LUminometric-based Methylation Assay (LUMA), dot blots and methylated DNA immunoprecipitation (MeDIP) analysis using previously described protocols. The complete methodologies are provided in the supplementary section.

\section{Western blot analysis; Gene expression analysis; Flow- cytometry analyses; Bio-informatic analysis}

Methods are presented in details in the supplementary section.

\section{Acknowledgements}

We would like to thank colleagues at UMR7216 'Epigenetics and Cell Fate' and at Institut Cochin (INSERM U1016, CNRS UMR8104, Univ. Paris Descartes) for helpful comments and suggestions during the conduct of this study. M.D. thank the DIM Biothérapies (Région Ile-de-France) and the Fondation pour la Recherche Médicale for postdoctoral fellowships. C.M. is a recipient of a MESRT PhD fellowship and Fondation pour la Recherche Médicale fellowship (FDT20150532354). We are embedded to Ms Kamelia Chebbout for performing some of the experiments. B.M. thank Laetitia Cointement for excellent administrative assistance and the scientists from the CYBIO and GENOM'IC platforms at Institut Cochin for help and trainings with cytometry and qPCR procedures and machines. Laboratories of B.M. and P.-A.D. are partners of Labex "Who am I?" (ANR-11-LABX-0071 and ANR-11-IDEX-005-02). This work was supported by grants from Electricité de France (\#123518) and Ligue Contre le Cancer - Comité de Paris (RS11/75-81; RS12/75/95-21; RS13/75-59). Work of B.M. is supported by FP7 Marie Curie action (PIRG07-GA-2010-268448), Fondation pour la Recherche Médicale (AJE20151234749), Institut National du Cancer-Plan Cancer (ASC15018KSA) and Institut Cochin (POC2016). PAD was supported by grants from Fondation ARC (Projet 2014), Ligue contre le cancer - Comité de Paris, and Institut National du Cancer (PLBIO 2015_1-PLBIO-01DRA-1). ML and STOS are supported by Fundação de Amparo à Pesquisa do Estado de São Paulo (FAPESP)

\section{Author details}

${ }^{1}$ INSERM, U1016, Institut Cochin, Paris, France. ${ }^{2}$ CNRS, UMR8104, Paris, France. ${ }^{3}$ Université Paris Descartes, Sorbonne Paris Cité, Paris, France. ${ }^{4}$ Université Paris Diderot, Sorbonne Paris Cité, Epigenetics and Cell Fate, UMR 7216 CNRS, 75013 Paris, France. ${ }^{5}$ Hematology and Blood Transfusion Center-University of Campinas/Hemocentro-Unicamp, Instituto Nacional de Ciência e Tecnologia do Sangue, Campinas, Brazil. ${ }^{6}$ Department of Biological Sciences, Federal University of São Paulo, Diadema, Brazil. ${ }^{7}$ Present address: Department of Biological Science, Florida State University, Tallahassee, FL 32306-4295, USA

\section{Author contributions}

B.M. designed the experiments. B.M., C.M., C.S.R., L.F., M.D., and N.G. performed the experiments and analyzed the data. M.L. and S.S. analyzed A.M.L. and M.D.S clinical data. B.M., C.M. and P.A.D. wrote the manuscript.
Conflict of interest

The authors declare no conflict of interest.

\section{Publisher's note}

Springer Nature remains neutral with regard to jurisdictional claims in published maps and institutional affiliations.

Supplementary Information accompanies this paper at (https://doi.org/ 10.1038/s41389-018-0092-0).

Received: 4 August 2018 Accepted: 22 August 2018

Published online: 11 October 2018

\section{References}

1. Fandy, T. E. et al. Early epigenetic changes and DNA damage do not predict clinical response in an overlapping schedule of 5-azacytidine and entinostat in patients with myeloid malignancies. Blood 114, 2764-2773 (2009).

2. Hagemann, S., Heil, O., Lyko, F. \& Brueckner, B. Azacytidine and decitabine induce gene-specific and non-random DNA demethylation in human cancer cell lines. PlOS ONE 6, e17388 (2011).

3. Hollenbach, P. W. et al. A comparison of azacitidine and decitabine activities in acute myeloid leukemia cell lines. PloS ONE 5, e9001 (2010).

4. Navada, S. C., Steinmann, J., Lübbert, M. \& Silverman, L. R. Clinical development of demethylating agents in hematology. J. Clin. Invest. 124, 40-46 (2014).

5. Fenaux, P. Inhibitors of DNA methylation: beyond myelodysplastic syndromes. Nat. Clin. Pract. Oncol. 2(Suppl 1), S36-S44 (2005).

6. Bohl, S. R., Bullinger, L. \& Rücker, F. G. Epigenetic therapy: azacytidine and decitabine in acute myeloid leukemia. Expert Rev. Hematol. 11, 361-371 (2018).

7. Sato, T., Issa, J.-P. \& Kropf, P. DNA hypomethylating drugs in cancer therapy. Cold Spring Harb Perspect Med. 7, a026948 (2017).

8. Treppendahl, M. B., Kristensen, L. S. \& Grønbæk, K. Predicting response to epigenetic therapy. J. Clin. Invest. 124, 47-55 (2014).

9. Unnikrishnan, A. et al. Integrative genomics identifies the molecular basis of resistance to Azacitidine Therapy in Myelodysplastic Syndromes. Cell Rep. 20, 572-585 (2017).

10. Tobiasson, $M$. et al. Mutations in histone modulators are associated with prolonged survival during azacitidine therapy. Oncotarget 7, 22103-22115 (2016).

11. Metzeler, K. H. et al. DNMT3A mutations and response to the hypomethylating agent decitabine in acute myeloid leukemia. Leukemia 26, 1106-1107 (2012).

12. Bally, C. et al. Prognostic value of TP53 gene mutations in myelodysplastic syndromes and acute myeloid leukemia treated with azacitidine. Leuk. Res. $\mathbf{3 8}$ 751-755 (2014)

13. Bejar, R. et al. TET2 mutations predict response to hypomethylating agents in myelodysplastic syndrome patients. Blood 124, 2705-2712 (2014).

14. Traina, F. et al. Impact of molecular mutations on treatment response to DNMT inhibitors in myelodysplasia and related neoplasms. Leukemia $\mathbf{2 8}$, 78-87 (2014)

15. Chiappinelli, K. B. et al. Inhibiting DNA methylation causes an interferon response in cancer via dsRNA including endogenous retroviruses. Cell 162, 974-986 (2015).

16. Duncavage, E. J. et al. Mutational landscape and response are conserved in peripheral blood of AML and MDS patients during decitabine therapy. Blood 2017.

17. Welch, J. S. et al. TP53 and Decitabine in Acute Myeloid Leukemia and Myelodysplastic Syndromes. N. Engl. J. Med. 375, 2023-2036 (2016).

18. Solly, F. et al. A miRNAs-DNMT1 axis is involved in azacitidine-resistance and predicts survival in higher risk myelodysplastic syndrome and low blast count acute myeloid leukemia. Clin Cancer Res. 23, 3025-3034 (2017).

19. Cluzeau, T. et al. BCL2L10 is a predictive factor for resistance to azacitidine in MDS and AML patients. Oncotarget 3, 490-501 (2012).

20. Follo, M. Y. et al. Reduction of phosphoinositide-phospholipase C beta1 methylation predicts the responsiveness to azacitidine in high-risk MDS. Proc. Natl Acad. Sci. USA 106, 16811-16816 (2009).

21. Linnekamp, J. F., Butter, R., Spijker, R., Medema, J. P. \& van Laarhoven, H. W. M. Clinical and biological effects of demethylating agents on solid tumours - A systematic review. Cancer Treat. Rev. 54, 10-23 (2017). 
22. Flotho, C. et al. The DNA methyltransferase inhibitors azacitidine, decitabine and zebularine exert differential effects on cancer gene expression in acute myeloid leukemia cells. Leukemia 23, 1019-1028 (2009).

23. Hsieh, Y.-Y. et al. Systematic discovery of drug action mechanisms by an integrated chemical genomics approach: identification of functional disparities between azacytidine and decitabine. Oncotarget 7, 27363-27378 (2016).

24. Leonova, K. I. et al. p53 cooperates with DNA methylation and a suicidal interferon response to maintain epigenetic silencing of repeats and noncoding RNAs. Proc. Natl Acad. Sci. USA 110, E89-E98 (2013).

25. Roulois, D. et al. DNA-demethylating agents target colorectal cancer cells by inducing viral mimicry by endogenous transcripts. Cell 162, 961-973 (2015).

26. Filion, G. J. P. et al. A family of human zinc finger proteins that bind methylated DNA and repress transcription. Mol. Cell Biol. 26, 169-181 (2006).

27. Sasai, N., Nakao, M. \& Defossez, P.-A. Sequence-specific recognition of methylated DNA by human zinc-finger proteins. Nucleic Acids Res. 38, 5015-5022 (2010).

28. Pozner, A. et al. The C-Terminal Zinc Fingers of ZBTB38 are Novel Selective Readers of DNA Methylation. - PubMed - NCBI. J. Mol. Biol. 430, 258-271 (2018).

29. Kotoku, T. et al. CIBZ regulates mesodermal and cardiac differentiation of by suppressing T and Mesp1 expression in mouse embryonic stem cells. Sci. Rep. 6, 34188 (2016).

30. Oikawa, Y. et al. The methyl-CpG-binding protein CIBZ suppresses myogenic differentiation by directly inhibiting myogenin expression. Cell Res. 21, 1578-1590 (2011)

31. Miotto, B. et al. The RBBP6/ZBTB38/MCM10 axis regulates DNA replication and common fragile site stability. Cell Rep. 7, 575-587 (2014).

32. Miotto, B. et al. Stabilization of the methyl-CpG binding protein ZBTB38 by the deubiquitinase USP9X limits the occurrence and toxicity of oxidative stress in human cells. Nucleic Acids Res 46, 4392-4404 (2018).

33. Buck-Koehntop, B. A. \& Defossez, P.-A. On how mammalian transcription factors recognize methylated DNA. Epigenetics 8, 131-137 (2013).

34. Wrangle, J. et al. Alterations of immune response of Non-Small Cell Lung Cancer with Azacytidine. Oncotarget 4, 2067-2079 (2013).

35. Itzykson, R. et al. Impact of TET2 mutations on response rate to azacitidine in myelodysplastic syndromes and low blast count acute myeloid leukemias. Leukemia 25, 1147-1152 (2011)

36. Giovannini, $C$. et al. CDKN1C/P57 is regulated by the Notch target gene Hes 1 and induces senescence in human hepatocellular carcinoma. Am. J. Pathol. 181, 413-422 (2012)

37. Radujkovic, A. et al. Expression of CDKN1C in the bone marrow of patients with myelodysplastic syndrome and secondary acute myeloid leukemia is associated with poor survival after conventional chemotherapy. Int J. Cancer 139, 1402-1413 (2016).
38. Pozner, A., Terooatea, T. W. \& Buck-Koehntop, B. A. Cell-specific Kaiso (ZBTB33) regulation of cell cycle through cyclin D1 and cyclin E1. J. Biol. Chem. 291 24538-24550 (2016)

39. Roussel-Gervais, A. et al. Loss of the methyl-CpG-binding protein ZBTB4 alters mitotic checkpoint, increases aneuploidy, and promotes tumorigenesis. Cancer Res. 77, 62-73 (2017)

40. Weber, A. et al. Zbtb4 represses transcription of P21CIP1 and controls the cellular response to p53 activation. EMBO J. 27, 1563-1574 (2008).

41. Koh, D.-I. et al. KAISO, a critical regulator of p53-mediated transcription of CDKN1A and apoptotic genes. Proc. Natl Acad. Sci. USA 111, 15078-15083 (2014).

42. Eggermann, T. et al. CDKN1C mutations: two sides of the same coin. Trends Mol. Med. 20, 614-622 (2014).

43. Hoffmann, M. J., Florl, A. R., Seifert, H.-H. \& Schulz, W. A. Multiple mechanisms downregulate CDKN1C in human bladder cancer. Int J. Cancer 114, 406-413 (2005).

44. Kamura, T. et al. Degradation of p57Kip2 mediated by SCFSkp2-dependent ubiquitylation. Proc. Natl Acad. Sci. USA 100, 10231-10236 (2003).

45. Ma, Y. et al. CDKN1C negatively regulates RNA polymerase ॥ C-terminal domain phosphorylation in an E2F1-dependent manner. J. Biol. Chem. 285, 9813-9822 (2010).

46. Haferlach, T. et al. Clinical utility of microarray-based gene expression profiling in the diagnosis and subclassification of leukemia: report from the International Microarray Innovations in Leukemia Study Group. J. Clin. Oncol. 28, 2529-2537 (2010)

47. Di Giammartino, D. C. et al. RBBP6 isoforms regulate the human polyadenylation machinery and modulate expression of mRNAs with AU-rich 3' UTRs. Genes Dev. 28, 2248-2260 (2014).

48. Duquesnes, N. et al. p57(Kip2) knock-in mouse reveals CDK-independent contribution in the development of Beckwith-Wiedemann syndrome. $J$. Pathol. 239, 250-261 (2016).

49. Gudbjartsson, D. F. et al. Many sequence variants affecting diversity of adult human height. Nat. Genet. 40, 609-615 (2008).

50. Clayton, P. et al. Characterizing short stature by insulin-like growth factor axis status and genetic associations: results from the prospective, cross-sectional, epidemiogenetic EPIGROW study. J. Clin. Endocrinol. Metab. 98, E1122-E1130 (2013).

51. Barretina, J. et al. The Cancer Cell Line Encyclopedia enables predictive modelling of anticancer drug sensitivity. Nature 483, 603-607 (2012).

52. Drusbosky, L. et al. Computational drug treatment simulations on projections of dysregulated protein networks derived from the myelodysplastic mutanome match clinical response in patients. Leuk. Res. 52, 1-7 (2017)

53. Valencia, A. et al. Expression of nucleoside-metabolizing enzymes in myelodysplastic syndromes and modulation of response to azacitidine. Leukemia $\mathbf{2 8}$ 621-628 (2014). 